\title{
Changing Americans' Attitudes about Immigration: \\ Using Moral Framing to Bolster Factual Arguments
}

\author{
Jan G. Voelkel ${ }^{1}$, Mashail Malik ${ }^{2 *}$, Chrystal Redekopp ${ }^{1}$, and Robb Willer ${ }^{1}$ \\ 1: Stanford University \\ 2: Harvard University \\ * Corresponding author: Mashail Malik, Department of Government, Harvard University, 1737 \\ Cambridge St, Cambridge, MA 02138, USA; phone: 443-253-4252; email: \\ mashailmalik@fas.harvard.edu.Co-author contact information: jvoelkel@stanford.edu, \\ redekopp@stanford.edu,willer@stanford.edu.
}

The paper was published at the ANNALS of the American Academy of Political and Social Science. This paper is not the copy of record and may not exactly replicate the final, authoritative version of the article. Please cite as

Voelkel, J. G., Malik, M., Redekopp, C., \& Willer, R. (2022). Changing Americans' attitudes about immigration: Using moral framing to bolster factual arguments. The ANNALS of the American Academy of Political and Social Science, 700(1), 73-85.

doi:10.1177/00027162221083877 


\begin{abstract}
How can the effect of appeals on immigration attitudes be bolstered? Partisans' tendency to interpret facts consistent with their priors impedes evidence-based persuasion. Accordingly, most prior work finds that favorable information about the impact of immigration has little or no influence on policy preferences. Here we propose that appealing to moral values can bolster the persuasive power of information. Across three experiments (total $N=4,616$ ), we find that an argument based on the value of in-group loyalty, which emphasized that immigrants are critical to America's economic strength, combined with information about the economic impact of legal immigration, significantly increased Americans' support for legal immigration. Additionally, we found a significant effect of the moral component of this message, whereas the effect of the information alone was of similar size but only marginally significant. These results show that moral arguments can strengthen the persuasiveness of informational appeals.
\end{abstract}

Keywords: immigration, persuasion, morality, information, moral reframing 
In the last decade, heated debates over immigration policy have featured prominently on the American political landscape. Many Americans and Western Europeans view immigration as one of the most important problems facing their country (Blinder 2015; Jones 2019), with more people favoring fewer new immigrants over allowing more to enter (Connor and Krogstad 2018). Accordingly, a vast social science literature has emerged to try and uncover the determinants of these attitudes. The thrust of this literature is that at least two types of concerns are important in shaping opposition to immigration: concerns about the negative economic impact of immigration, and concerns about the cultural changes that accompany immigration (for a review, see Hainmueller and Hopkins 2014).

However, not all these concerns correspond to facts about the real-world impacts of immigration or the true characteristics of immigrant populations. There is mounting evidence that immigration is a net positive for the American economy, and that immigrants often go to great lengths to assimilate to the American way of life (Hathaway 2017; Passell and Cohn 2017; Myers et al. 2013; National Academies of Sciences, Engineering, and Medicine 2015, 2017). Yet, citizens are often dramatically misinformed about the characteristics and size of immigrant communities (Blinder 2015; Citrin and Sides 2008). If such misperceptions are a driver of anti-immigration attitudes, can disseminating factual information help shape policy preferences in a proimmigration direction?

Like for many other highly polarized issues, the answer appears to be a tentative "no." Empirical tests of informational treatments provide mixed results, with more evidence against their effectiveness than for. Several studies found that providing accurate information about the characteristics and effects of immigrant populations does not meaningfully impact policy preferences, even when respondents update their knowledge in response to factual information 
(Barrera et al. 2020; Grigorieff, Roth and Ubfal 2020; Hopkins, Sides and Citrin 2019; Jorgensen and Osmundsen 2020). One explanation is that for issues tied to a strongly held identity -- such as political partisanship -- individuals are motivated to discount new information that threatens the identity and associated worldviews (Ecker et al. 2014; Jerit and Zhao 2020; Leeper and Slothuus 2014).

In the face of such resistance, how can we increase the uptake and persuasiveness of factual information? In this paper, we propose that one way is to couch facts within frames that resonate with the recipient's values. We show across three separate studies $(n=4,546)$ that communicating accurate information about the economic benefits of immigration within a value frame appealing to patriotism and group loyalty is effective in changing immigration policy preferences. These results are in line with other value-framing interventions that have successfully shifted opinions on similarly contentious issues such as climate change (Feinberg and Willer 2013; Wolsko et al. 2016), same-sex marriage (Feinberg and Willer 2015), and political campaigns (Voelkel and Feinberg 2018; Voelkel, Mernyk and Willer 2021) and suggest that moral framing is an effective tool for opening people up to accepting science-based information.

\section{Informational Interventions and Attitude Change}

Why might providing accurate, positive information about immigrants affect the public's willingness to accept more immigration? One reason is that opposition to immigration might be caused by widespread misperceptions stemming from misinformation campaigns. Opponents of immigration often spread negative misinformation about immigrant populations (Humprecht 2019). Accordingly, citizens' perceptions of immigrant characteristics often differ from official data, and these misperceptions are correlated with immigration policy preferences (Blinder 2015; Zhirkov 2020). Opposition to immigration is associated with concerns about the possible negative 
economic and/or cultural impacts of immigration (Hainmueller and Hopkins 2014). Positive and accurate information about immigration's impacts might help combat these concerns.

A handful of studies have tested informational interventions, with limited success. Hopkins, Sides and Citrin (2019) show in seven separate surveys conducted over a span of 11 years that correcting misperceptions about the size of immigrant populations (which opponents tend to overestimate) has little impact on immigration policy preferences. Similarly, Jorgensen and Osmundsen (2020), Barrera et al. (2020) and Grigorieff, Roth and Ubfal (2020) find that while respondents update their beliefs about immigrant characteristics, informational treatments largely fail to change immigration policy preferences. In contrast, Abascal et al. (2021), Haaland and Roth (2020), and Facchini et al. (2016) find certain facts to be persuasive in increasing openness to immigration.

That immigration policy preferences are generally difficult to change through informational interventions is consistent with much of the literature on political misinformation (for a review, see Jerit and Zhao 2020). There is growing evidence that while people can update their beliefs in response to the specific factual information, they simultaneously tend to reinterpret this new information to fit their existing policy preferences (Bisgaard 2015; Nyhan et al. 2019; Thorson 2016). It is theorized that these effects can be explained by motivated reasoning, which is particularly operative in cases where the issue area is highly polarized or connected to an individual's strongly held identity (Ecker et al. 2014). Immigration is one such issue area (Daniller 2019), which may explain why informational interventions rarely change preferences in this policy realm.

\section{The Persuasiveness of Moral Frames}


How might we increase the persuasiveness of informational interventions that are designed to change policy preferences? A promising approach is moral reframing -- a persuasion technique in which advocacy for a policy position is framed in language that is consistent with the moral values of the audience (Feinberg and Willer 2019). Moral reframing interventions have successfully shifted opinions on contentious issues such as climate change (Feinberg and Willer 2013; Wolsko et al. 2016), same-sex marriage (Feinberg and Willer 2015), and political campaigns (Voelkel and Feinberg 2018; Voelkel, Mernyk and Willer 2021). For example, reframing arguments about climate change by using language consistent with conservative values (e.g., purity and patriotism) leads conservatives to become more supportive of pro-environmental policies (Feinberg and Willer 2013; Feygina et al. 2010; Wolsko et al. 2016).

The assumption behind moral reframing interventions is that moral convictions are an important basis for people's attitudes and policy preferences (Feinberg and Willer 2019). Importantly, in this approach, facts take a backseat to the fit between issue positions and individuals' moral values. If motivated reasoning occurs in the face of factual information due to a perceived threat to one's identity or self-concept, for example, then couching such information in a frame that appeals to one's fundamental convictions may increase the persuasiveness of the argument. In the realm of immigration preferences, appealing to moral values might help close the gap between updating beliefs about immigration and updating associated policy preferences. ${ }^{1}$

\section{The Current Research}

We conducted three experiments to test whether couching accurate information about immigration within a compelling moral frame can increase Americans' support for legal immigration. All studies were approved by the Institutional Review Board at Stanford University.

\footnotetext{
${ }^{1}$ Relatedly, Simonsen and Bonikowski (2021) find that morally charged anti-immigrant rhetoric can cause more affective polarization, but does not produce more extreme attitudes on immigration.
} 
All participants provided informed consent. Materials, anonymized data (including descriptions of how the original files were anonymized), and analysis code for Studies 1 - 3 are available via https://osf.io/ympkz/. Preregistrations for the analysis plan in Study 1 and for the hypothesis and analysis plan in Study 3 are available via https://osf.io/fexvn (Study 1) and https://osf.io/px3d9 (Study 3).

\section{Study 1}

Study 1 provides an initial test of whether moral frames influence Americans' support for legal immigration. We tested three different frames that included the most common arguments discussed in the immigration literature: economic concerns, cultural concerns, and humanitarian concerns. ${ }^{2}$ We embedded facts from these three domains in arguments based on loyalty values (for the economic and cultural effects) or minimizing harm values (for the humanitarian effects). Based on the moral reframing literature (e.g., Feinberg and Willer 2019), we expected a frame condition $\mathrm{x}$ ideology interaction effect, such that the loyalty frames would be more effective among conservative participants whereas the reducing harm-humanitarian frame would be more effective among liberal participants.

\section{Methods}

We recruited US residents from a large panel of previously surveyed Amazon Mechanical Turk workers ( $n=906$; for the exclusion rules and sample demographics, see Tables S1 and S2 in the SI). The sample size provides us with 80 percent power to detect treatment effects of Cohen's $f \geq 0.03$ and with 95 percent power to detect treatment effects of Cohen's $f \geq 0.04$. This study had

\footnotetext{
${ }^{2}$ While our focus is on how morally framed information on the economic or cultural impacts of immigration influences attitudes, we also explored the effect of morally framed information on the suffering alleviating impacts of immigration. Prior work suggests that invoking empathy increases openness to refugees (Adida, Lo and Platas 2018).
} 
a 4 (frame condition: control vs loyalty-economy vs loyalty-culture vs harm-humanitarian; between-subjects) x 2 (time: pre-treatment vs post-treatment; within-subjects) design.

We conducted the pre-treatment survey in December 2018 to measure our main dependent variable, participants' support for legal immigration. Support for legal immigration was measured with two items ${ }^{3}\left(r_{\text {Spearman-Brown }}=0.92\right)$. The two items were measured on seven-point scales ranging from "Be decreased a lot" to "Be increased a lot": (i) "Do you think the number of legal immigrants coming to the United States each year should be increased, reduced or remain the same? By legal immigration we mean people legally coming to the United States from other countries to live here" and (ii) "Do you think that the number of green cards available for immigrants coming to the United States each year should be increased, reduced or remain the same?". In this and all following surveys, we also collected demographic variables and some additional dependent variables (see https://osf.io/ympkz/), with analyses available in the Supplementary Materials.

Approximately one week later, we contacted the participants who completed the first survey to participate in a second survey. Participants were randomly assigned to one of four experimental conditions: the control condition, the loyalty-economy frame condition, the loyaltyculture frame condition, or the harm-humanitarian frame condition. In the control frame condition, participants read an essay about the history of neckties. In the loyalty-economy frame condition, participants read several facts about the economic effects of immigration couched within a frame emphasizing moral values such as showing loyalty and patriotism. In the loyalty-culture frame condition, participants read several facts about the cultural effects of immigration couched within a frame emphasizing moral values such as showing loyalty and patriotism. In the harm-

\footnotetext{
${ }^{3}$ In Study 1, we preregistered to use only the first item as our main dependent variable. However, because the two items were very strongly correlated, we decided to use a composite of these two items instead. This strategy was preregistered in the analysis plan for Study 3. We report the results for the individual items in the Supplementary Materials.
} 
humanitarian frame condition, participants read several facts about the humanitarian effects of immigration couched within a frame emphasizing moral values such as providing care and reducing harm. The full text of all essays is available in the SI. Afterwards, we measured participants' support for legal immigration using the same two-item measure as in the first survey

$\left(r_{\text {Spearman-Brown }}=0.94\right)$.

\section{Results and Discussion}

We tested the effects of the treatments on support for legal immigration using linear regression analyses, in which we controlled for participants' gender, age, race, education, and ideology. We recoded our measure of support for legal immigration to range from 0 to 1 . We used the difference of the post-treatment measure and the pre-treatment measure as our dependent variable. The results are illustrated in Figure 1. We used $p<.05$ as our inference 
Figure 1: Effects of the Frame Condition on Change in Support for Legal Immigration, Study 1

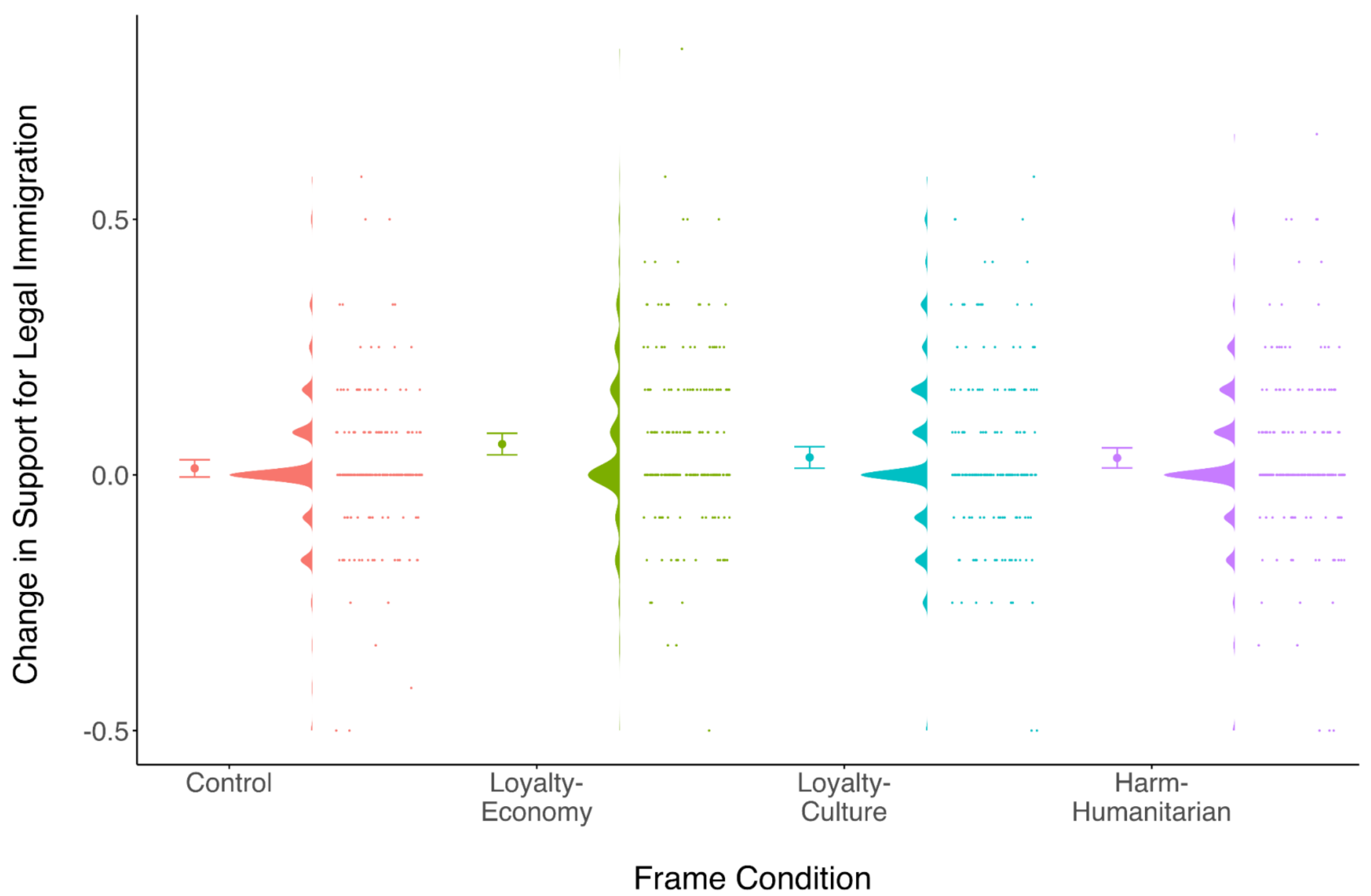


criterion. We conducted Holm-Bonferroni adjustment for multiple hypothesis testing as a (nonpreregistered) robustness check.

The loyalty-economy frame increased support for legal immigration. Participants in the loyalty-economy frame condition $\left(M_{\text {Pre }}=0.48, S D_{\text {Pre }}=0.25 ; M_{\text {Post }}=0.54, S D_{\text {Post }}=0.24\right)$ became significantly more supportive of legal immigration than participants in the control condition $\left(M_{\text {Pre }}\right.$ $\left.=0.47, S D_{\text {Pre }}=0.26 ; M_{\text {Post }}=0.48, S D_{\text {Post }}=0.25\right), b=0.05, S E=0.01, t(891)=3.19, p=.001, p_{\text {HBA }}$ $=.004$, Cohen's $d=0.30$.

The loyalty-culture frame did not increase support for legal immigration. Participants in the loyalty-culture frame condition $\left(M_{\text {Pre }}=0.52, S D_{\text {Pre }}=0.25 ; M_{\text {Post }}=0.55, S D\right.$ Post $\left.=0.26\right)$ did not become significantly more or less supportive of legal immigration than participants in the control condition $\left(M_{\text {Pre }}=0.47, S D_{\text {Pre }}=0.26 ; M_{\text {Post }}=0.48, S D_{\text {Post }}=0.25\right), b=0.02, S E=0.01, t(891)=$ $1.43, p=.153, p$ HBA $=.307$, Cohen's $d=0.13$.

The harm-humanitarian frame did not increase support for legal immigration. Participants in the harm-humanitarian frame condition $\left(M_{\text {Pre }}=0.50, S D\right.$ Pre $=0.25 ; M$ Post $=0.53, S D$ Post $\left.=0.26\right)$ did not become significantly more or less supportive of legal immigration than participants in the control condition $\left(M_{\text {Pre }}=0.47, S D\right.$ Pre $=0.26 ; M_{\text {Post }}=0.48, S D$ Post $\left.=0.25\right), b=0.02, S E=0.01, t(891)$ $=1.28, p=.200, p_{\text {HвA }}=.307$, Cohen's $d=0.12$.

We did not find evidence for a frame condition x political ideology interaction effect, $F(6$, $885)=0.60, p=.733, \Delta R^{2}=0.00$.

Study 1 provides initial evidence that combining facts about the impact of immigration on the economy with a moral argument that supporting legal immigration is patriotic increases people's support for legal immigration. The results were inconclusive for the effects of a loyalty- 
culture frame and a harm-humanitarian frame. Surprisingly, we did not find evidence that the frames had different effects among different ideological groups.

\section{Study 2}

Study 2 was a replication of Study 1, except in that we dropped the repeated measurement part of the design.

\section{Methods}

We recruited a new sample of US residents from the same population as in Study 1 ( $n=$ 1640; for the exclusion rules and sample demographics, see Tables S1 and S2 in the SI). The sample size provides us with 80 percent power to detect treatment main effects of Cohen's $d \geq$ 0.20 and with 95 percent power to detect treatment main effects of Cohen's $d \geq 0.25$. This study had a between-subjects design with four frame conditions (control vs loyalty-economy vs loyaltyculture vs harm-humanitarian).

We first collected some potential moderators. Next, participants were randomly assigned to one of the same four experimental conditions from Study 1. We used the same treatment texts as in Study 1. Afterwards, we measured participants' support for legal immigration using the same two-item measure as in Study $1(r$ Spearman-Brown $=0.92)$.

\section{Results and Discussion}

We used the same analytical strategy as in Study 1, except that we only had a post-treatment measure of support for legal immigration, which served as our dependent variable instead of the difference score. The results are illustrated in Figure 2. 
Figure 2: Effects of the Frame Condition on Support for Legal Immigration, Study 2

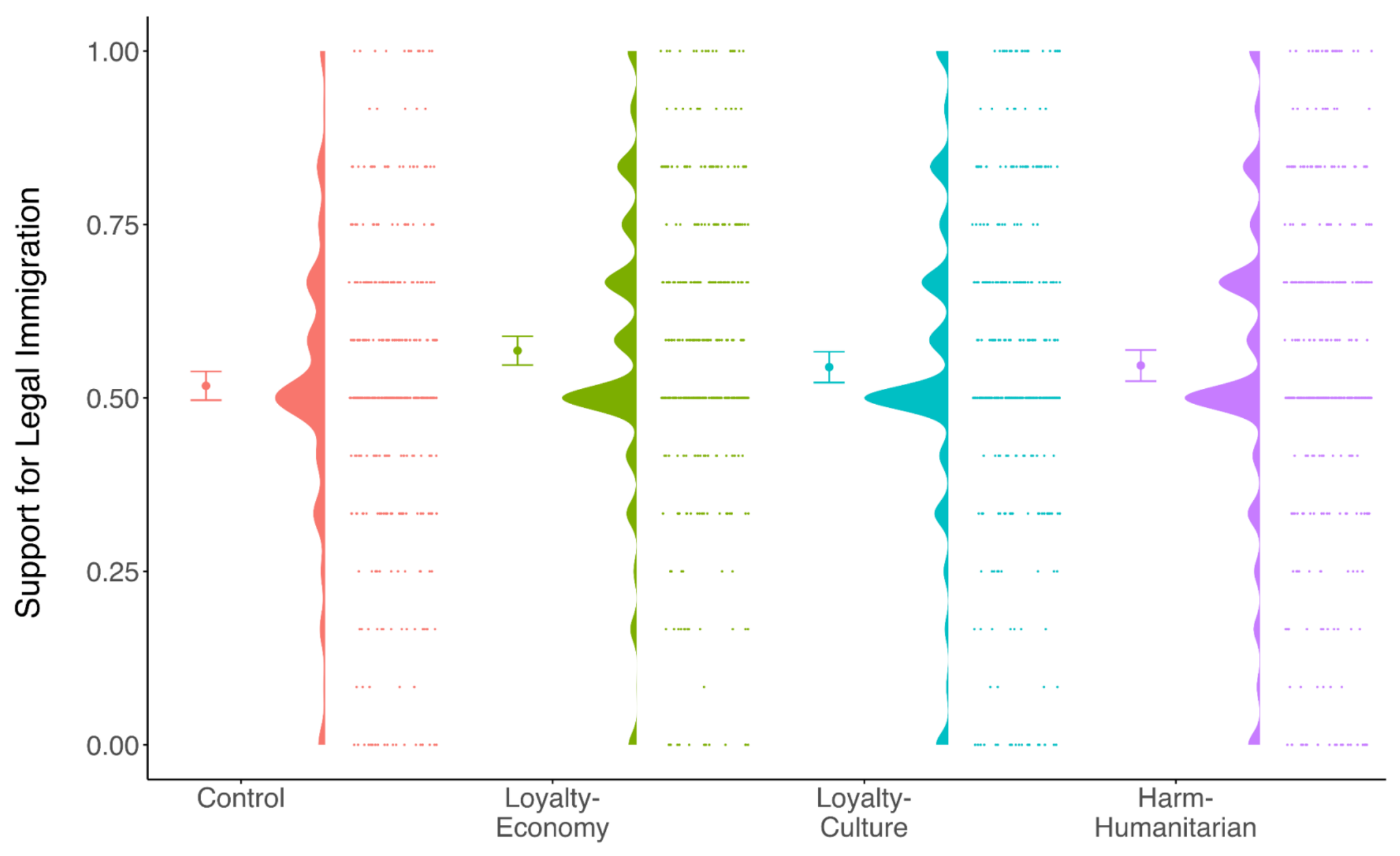

Frame Condition 
The loyalty-economy frame increased support for legal immigration. Participants in the loyalty-economy frame condition $(M=0.57, S D=0.21)$ reported significantly higher support for legal immigration than participants in the control condition $(M=0.52, S D=0.21), b=0.05, S E=$ $0.01, t(1622)=3.52, p<.001, p_{\mathrm{HBA}}=.001$, Cohen's $d=0.23$.

The loyalty-culture frame also increased support for legal immigration. Participants in the loyalty-culture frame condition $(M=0.54, S D=0.23)$ reported significantly higher support for legal immigration than participants in the control condition $(M=0.52, S D=0.21), b=0.04, S E=$ $0.01, t(1622)=2.49, p=.013, p_{\mathrm{HBA}}=.026$, Cohen's $d=0.16$.

The harm-humanitarian frame also increased support for legal immigration. Participants in the harm-humanitarian frame condition $(M=0.55, S D=0.23)$ reported significantly higher support for legal immigration than participants in the control condition $(M=0.52, S D=0.21), b=0.03$, $S E=0.01, t(1622)=2.04, p=.041, p_{\mathrm{HBA}}=.041$, Cohen's $d=0.13$.

We did not find evidence for a frame condition x political ideology interaction effect, $F(6$, 1616) $=1.17, p=.322, \Delta R^{2}=0.00$.

Study 2 provides additional evidence that combining facts about the impact of immigration on the economy with a moral argument that supporting legal immigration is patriotic increases people's support for legal immigration. In this study, we also found significant effects of a loyaltyculture frame and a harm-humanitarian frame. As in Study 1, we did not find evidence that the frames had different effects among different ideological groups.

\section{Study 3}

Study 3 had two goals. The first goal was to replicate the effect of the frame with the strongest and most consistent effect on support for legal immigration, the loyalty-economy frame. We preregistered this hypothesis and our analysis plan. The second goal was to examine which 
components of this treatment are necessary for persuasion, since the loyalty-economy frame is a bundled treatment combining accurate information with moral language. Therefore, we created four conditions: a condition with both facts and moral language, a condition with only the facts, a condition with only the moral language, and a control condition. This design allows us to identify whether facts, moral framing, or both are needed to shape Americans' support for legal immigration.

\section{Methods}

Our goal was to recruit 2000 participants. This target was based on a priori power analyses with $\mathrm{G}^{*}$ Power, suggesting this would give us $95 \%$ power to detect the effect size of Cohen's $\mathrm{d}=$ 0.23 that we found in Study 2. We recruited a new sample of US residents from the same population as in Studies 1 and 2 ( $n=2070$; for the exclusion rules and sample demographics, see Tables S1 and S2 in the SI). This sample size provides us with 80 percent power to detect treatment main effects of Cohen's $d \geq 0.17$ and with 95 percent power to detect treatment main effects of Cohen's $d \geq 0.22$. This study had a between-subjects design with four frame conditions (control vs economic facts and loyalty frame vs loyalty frame alone vs economic facts alone).

We first collected some potential moderators. Next, participants were randomly assigned to one of four experimental conditions: the control condition, the economic facts and loyalty frame condition, the loyalty frame alone condition, or the economic facts alone condition. The control condition featured the same essay about the history of neckties as in Studies 1 and 2. In the economic facts alone condition, participants read about accurate information about the economic effects of immigration (without a moral frame). In the loyalty frame alone condition, participants read a frame connecting supporting legal immigration with moral values such as showing loyalty and patriotism (without facts). In the economic facts and loyalty frame condition, participants read 
both the information about the economic effects of immigration and the moral frame. The full text of all essays is available in the SI. Afterwards, we measured participants' support for legal immigration using the same measure as in Studies 1 and $2(r$ Spearman-Brown $=0.93)$.

\section{Results and Discussion}

We used the same analytical strategy as in Study 2. The results are illustrated in Figure 3.

The economic facts and loyalty frame condition increased support for legal immigration. Participants in the economic facts and loyalty frame condition $(M=0.59, S D=0.22)$ reported significantly higher support for legal immigration than participants in the control condition $(M=$ $0.55, S D=0.23), b=0.04, S E=0.01, t(2052)=2.92, p=.004, p$ HBA $=.011$, Cohen's $d=0.17$.

The loyalty frame alone condition did not increase support for legal immigration. Participants in the loyalty frame alone condition $(M=0.58, S D=0.22)$ reported marginally significantly higher support for legal immigration than participants in the control condition $(M=$ $0.55, S D=0.23), b=0.03, S E=0.01, t(2052)=1.95, p=.051$, Cohen's $d=0.11$. Note that this effect was no longer marginally significant after a Holm-Bonferroni adjustment for multiple hypothesis testing $\left(p_{\mathrm{HBA}}=.102\right)$. 
Figure 3: Effects of the Frame Condition on Support for Legal Immigration, Study 3

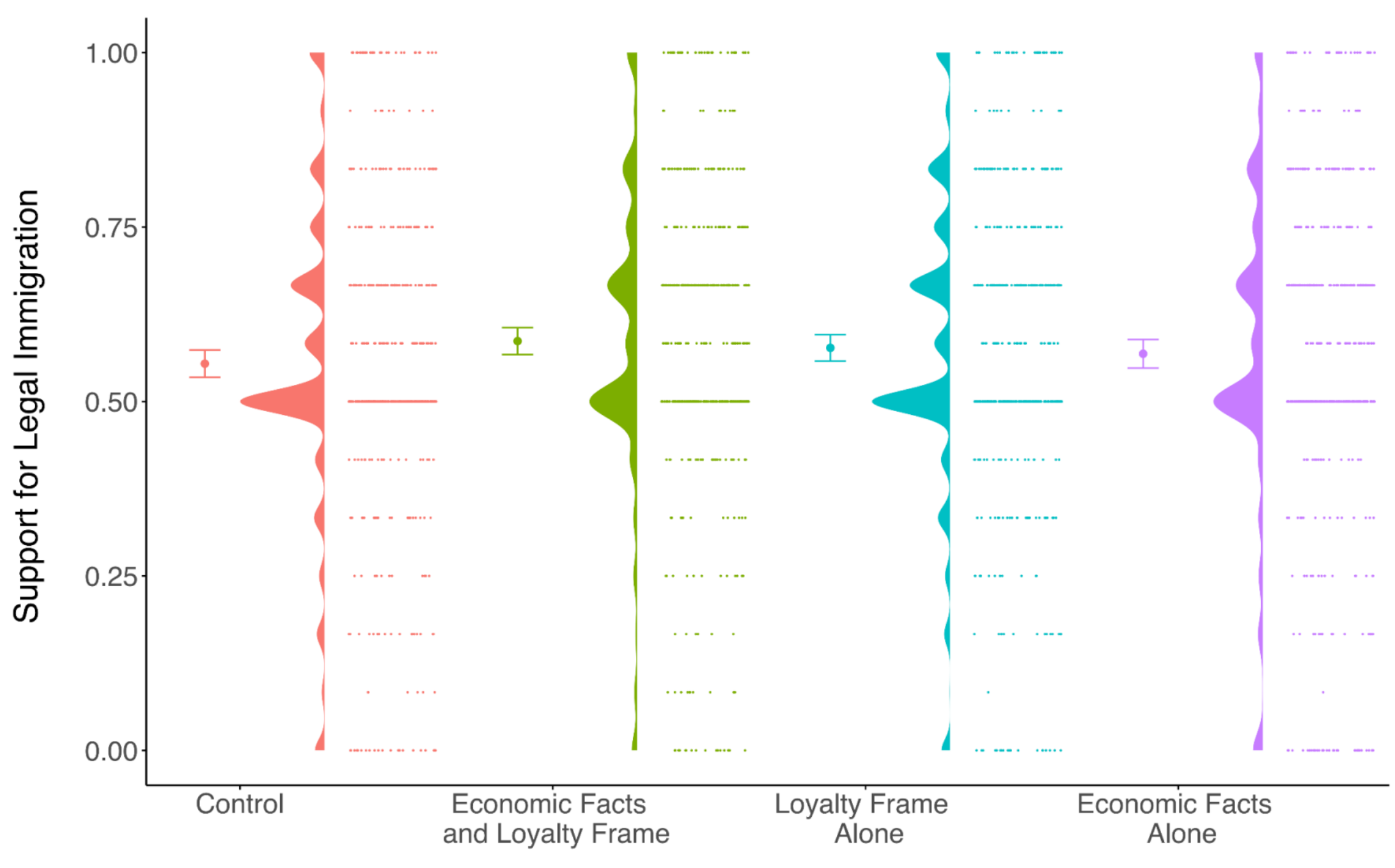

Condition 
The economic facts alone condition did not increase support for legal immigration. Participants in the economic facts alone condition $(M=0.57, S D=0.24)$ did not report significantly higher or lower support for legal immigration than participants in the control condition $(M=0.55$, $S D=0.23), b=0.02, S E=0.01, t(2052)=1.58, p=.115, p_{\text {HBA }}=.115$, Cohen's $d=0.09$.

As in the previous two studies, we did not find evidence for a frame condition x political ideology interaction effect, $F(6,2046)=0.64, p=.700, \Delta R^{2}=0.00$.

Does the effect of economic facts depend on being paired with the loyalty frame or do these two message components have independent effects? We sought to answer this question by treating our study as a 2 (read economic facts: yes vs no) x 2 (read loyalty frame: yes vs no) design. We found that participants who read the loyalty frame $(M=0.58, S D=0.22)$ reported significantly higher support for legal immigration than participants who did not read the loyalty frame $(M=$ $0.56, S D=0.23), b=0.02, S E=0.01, t(2053)=2.34, p=.020$, Cohen's $d=0.09$. We found that participants who read the economic facts $(M=0.58, S D=0.23)$ reported marginally significantly higher support for legal immigration than participants who did not read the economic facts $(M=$ $0.57, S D=0.22), b=0.02, S E=0.01, t(2053)=1.80, p=.072$, Cohen's $d=0.07$. We did not find evidence for a loyalty frame $\mathrm{x}$ economic facts interaction effect, $F(1,2052)=0.18, p=.671, \Delta R^{2}$ $=0.00$.

Study 3 provides additional evidence that combining facts about the impact of immigration on the economy with a moral argument that supporting legal immigration is patriotic increases people's support for legal immigration. Although the non-significant results for the interaction effect of the two message components do not imply evidence for a null effect, our results seem to be more consistent with two independent effects of the moral frame and economic facts (although the latter was only marginally significant). We recommend that future research test the 
independent effects of moral and facts-based arguments with the large samples needed to identify potentially small effects. For now, we conclude that the combination of moral framing and facts can persuade people on the topic of immigration and that moral framing seems to increase the persuasiveness of factual arguments.

\section{General Discussion}

The studies above depart from extant informational interventions by providing accurate information about immigrant populations within a moral frame. The results suggest that the persuasiveness of positive information about the economic effects of immigration is bolstered when couched in moral language based on the value of group loyalty.

Importantly, our treatment effects are not moderated by political ideology (nor other political variables such as partisanship; see Supplementary Materials). This result differs from prior work on moral reframing (Feinberg and Willer 2019). One potential explanation is that our treatment draws on civic (as opposed to ethnic) patriotism. While some forms of exclusionary patriotism (or nationalism) may resonate more with conservative audiences, a majority of Americans across the partisan divide consider themselves patriotic (Population Research Institute 2019). Our research suggests that leveraging civic patriotism resonates across the ideological spectrum and can bypass psychological biases (such as motivated reasoning) that keep audiences from updating their beliefs and/or associated policy preferences. Future work should explore other such moral frames that advocacy campaigns can leverage to increase the persuasiveness of their messaging and to reduce polarization.

Finally, we want to emphasize that moral framing about immigration is not intrinsically a good or bad thing to do. As in other contexts, moral framing can be misused if actors are disingenuous or manipulative. Our research suggests that the link between the topic of immigration 
and different moral values is malleable. Thus, activists and politicians might be well advised to think about what arguments make the most moral sense to their audience. 


\section{References}

Abascal, Maria, Tiffany J. Huang, and Van C. Tran. 2021. Intervening in anti-immigrant sentiments: the causal effects of factual information on attitudes toward immigration.” The ANNALS of the American Academy of Political and Social Science.

Adida, Claire L., Adeline Lo, and Melina R. Platas. 2018. Perspective taking can promote shortterm inclusionary behavior toward Syrian refugees. Proceedings of the National Academy of Sciences 115:9521-9526.

Barrera, Oscar, Sergei Guriev, Emeric Henry, and Ekaterina Zhuravskaya. 2020. Facts, alternative facts, and fact checking in times of post-truth politics. Journal of Public Economics 182:104-123.

Bisgaard, Martin. 2015. Bias will find a way: Economic perceptions, attributions of blame, and partisan-motivated reasoning during crisis. The Journal of Politics 77:849-860.

Blinder, Scott. 2015. Imagined immigration: The impact of different meanings of 'immigrants' in public opinion and policy debates in Britain. Political Studies 63:80-100.

Citrin, Jack, and John Sides. 2008. Immigration and the imagined community in Europe and the United States. Political Studies 56:33-56.

Connor, Phillip, and Jens Manuel Krogstad. 2018. Many worldwide oppose more migration. Pew Research Center. https://tinyurl.com/mtahdu2x Daniller, Andrew. 2019. Americans' immigration policy priorities: Divisions between and within - the two parties. Pew Research Center. https://tinyurl.com/2tsnk7fz

Druckman, James N., and Mary C. McGrath. 2019. The evidence for motivated reasoning in climate change preference formation. Nature Climate Change 9:111-119. 
Ecker, Ullrich K. H., Stephan Lewandowsky, Olivia Fenton, and Kelsey Martin. 2014. Do people keep believing because they want to? Preexisting attitudes and the continued influence of misinformation. Memory \& Cognition 42:292-304.

Facchini, Giovanni, Yotam Margalit, and Hiroyuki Nakata. 2016. Countering public opposition to immigration: the impact of information campaigns. SSRN. https://papers.ssrn.com/abstract=2887349.

Feinberg, Matthew, and Robb Willer. 2013. The moral roots of environmental attitudes. Psychological Science 24:56-62.

Feinberg, Matthew, and Robb Willer. 2015. From gulf to bridge: when do moral arguments facilitate political influence? Personality and Social Psychology Bulletin 41:1665-1681. Feinberg, Matthew, and Robb Willer. 2019. Moral reframing: a technique for effective and persuasive communication across political divides. Social and Personality Psychology Compass 13:e12501.

Feygina, Irina, John T. Jost, and Rachel E. Goldsmith. 2010. System justification, the denial of global warming, and the possibility of 'system-sanctioned change.' Personality and Social Psychology Bulletin 36:326-338.

Grigorieff, Alexis, Christopher Roth, and Diego Ubfal. 2020. Does information change attitudes toward immigrants? Demography 57:1117-1143.

Haaland, Ingar, and Christopher Roth. 2020. Labor market concerns and support for immigration. Journal of Public Economics 191:104256.

Hainmueller, Jens, and Daniel J. Hopkins. 2014. Public attitudes toward immigration. Annual Review of Political Science 17:225-249. 
Hart, P. Sol, and Erik C. Nisbet. 2012. Boomerang effects in science communication: how motivated reasoning and identity cues amplify opinion polarization about climate mitigation policies. Communication Research 39:701-723.

Hathaway, Ian. 2017. Almost half of fortune 500 companies were founded by American immigrants or their children. Brookings. https://tinyurl.com/efdhjvzf

Hopkins, Daniel J., John Sides, and Jack Citrin. 2019. The muted consequences of correct information about immigration. The Journal of Politics 81:315-320.

Humprecht, Edda. 2019. Where 'fake news' flourishes: a comparison across four western democracies. Information, Communication \& Society 22:1973-1988.

Jones, Jeffrey M. 2019. New high in U.S. say immigration most important problem.” Gallup. https://tinyurl.com/k9hvy5m7

Jørgensen, Frederik Juhl, and Mathias Osmundsen. 2020. Correcting citizens’ misperceptions about non-western immigrants: corrective information, interpretations, and policy opinions. Journal of Experimental Political Science 1-10.

Kahan, Dan M. 2016. The politically motivated reasoning paradigm, part 1: What politically motivated reasoning is and how to measure it. In Emerging trends in the social and behavioral sciences: an interdisciplinary, searchable, and linkable resource, eds. Robert Scott and Stephen Kosslyn, 1-16. New York, NY: John Wiley \& Sons, Inc.

Lee, Michelle Ye Hee. 2015. Donald Trump's false comments connecting Mexican immigrants and crime. Washington Post. https://tinyurl.com/3mnb5ud3

Leeper, Thomas J., and Rune Slothuus. 2014. Political parties, motivated reasoning, and public opinion formation. Political Psychology 35:S1-129. 
Myers, Dowell, Stephen Levy, and John Pitkin. 2013. The contributions of immigrants and their children to the American workforce and jobs of the future. Center for American Progress. https://tinyurl.com/3xvb4xu2

National Academies of Sciences, Engineering, and Medicine. 2015. The Integration of Immigrants into American Society. Washington, DC: The National Academies Press. National Academies of Sciences, Engineering, and Medicine. 2017. The Economic and Fiscal Consequences of Immigration. Washington, DC: The National Academies Press.

Nyhan, Brendan, Ethan Porter, Jason Reifler, and Thomas J. Wood. 2020. Taking fact-checks literally but not seriously? The effects of journalistic fact-checking on factual beliefs and candidate favorability. Political Behavior 42:3-939.

Passell, Jeffrey S., and D’Vera Cohn. 2017. Immigration projected to drive potential U.S. labor force growth through 2035. Pew Research Center. https://tinyurl.com/nbyy3hax

Simonsen, Kristina, and Bart Bonikowski. 2021. Moralizing immigration: the impact of political framing on polarization in the United States and Denmark. Comparative Political Studies.

Thorson, Emily. 2016. Belief echoes: the persistent effects of corrected misinformation. Political Communication 33:3-460.

Population Research Institute. 2019. Though politically divided, Americans remain patriotic. https://tinyurl.com/56rnx6yf

Voelkel, Jan G., and Matthew Feinberg. 2018. Morally reframed arguments can affect support for political candidates. Social Psychological and Personality Science 9:8-917. 
Voelkel, Jan G., Joseph Mernyk, and Robb Willer. 2021. Navigating the progressive paradox: the effects of value reframing on support for economically progressive candidates. OSF Preprints. https://osf.io/preprints/socarxiv/kdjc5/

Wolsko, Christopher, Hector Ariceaga, and Jesse Seiden. 2016. Red, white, and blue enough to be green: effects of moral framing on climate change attitudes and conservation behaviors. Journal of Experimental Social Psychology 65-7.

Zhirkov, Kirill. 2021. Who are 'the immigrants'? Beliefs about immigrant populations and antiimmigration attitudes in the United States and Britain. Social Science Quarterly 102:1228. 